\title{
Conditional disruption of AMP kinase in dopaminergic neurons promotes Parkinson's disease-associated phenotypes in mice
}

\section{Liting Hang}

Nanyang Technological University

Geraldine W.Y. Goh

Nanyang Technological University

\section{Ziyin Wang}

National Neuroscience Institute

\section{Huey Ching Choong}

National Neuroscience Institute

John Thundyil

National Neuroscience Institute

Shengli Xu

A*STAR Bioprocessing Technology Institute

Kong Peng Lam

A*STAR Bioprocessing Technology Institute

Kah-Leong Lim ( $\sim$ kahleong.lim@ntu.edu.sg )

Nanyang Technological University https://orcid.org/0000-0002-5440-2588

\section{Short report}

Keywords: Parkinson's disease, AMPK, mitochondria

Posted Date: July 27th, 2020

DOI: https://doi.org/10.21203/rs.3.rs-43593/v1

License: (1) (1) This work is licensed under a Creative Commons Attribution 4.0 International License. Read Full License 
1 Conditional disruption of AMP kinase in dopaminergic neurons promotes

2 Parkinson's disease-associated phenotypes in mice

3

4

5

6 9

Liting Hang ${ }^{1,2,3,5}$, Geraldine W.Y. Goh ${ }^{1,5}$, Ziyin Wang ${ }^{5}$, Huey Ching Chong ${ }^{5}$, John Thundyil ${ }^{5}$, Shengli $\mathrm{Xu}^{3,6}$, Kong Peng, Lam ${ }^{4,6}$, Kah-Leong Lim ${ }^{1,5 *}$

${ }^{1}$ Lee Kong Chian School of Medicine, Nanyang Technological University Singapore, Singapore. ${ }^{2}$ NUS Graduate School for Integrative Sciences and Engineering, National University of Singapore, Singapore. ${ }^{3}$ Department of Physiology, ${ }^{4}$ Department of Microbiology \& Immunology, National University of Singapore, Singapore. ${ }^{5}$ Department of Research, National Neuroscience Institute, Singapore. ${ }^{6}$ A*STAR Bioprocessing Technology Institute, Singapore.

*To whom all correspondence should be addressed: Kah-Leong Lim, Ph.D., Lee Kong Chian School of Medicine, Nanyang Technological University Singapore. 11 Mandalay Road, Singapore 308232. Email: kahleong.lim@ntu.edu.sg

Email addresses:

liting.hang@ntu.edu.sg

geraldine.gohwy@ntu.edu.sg

ziyin_wang@nni.com.sg

huey_ching_choong@,nni.com.sg

leji.john@gmail.com

xu shengli@bti.a-star.edu.sg

lam_kong_peng@bti.a-star.edu.sg

(1)

8

9

(1)

Number of Figures and Tables: 4 figures; 1 Table

Number of pages/words: 23 pages/4466 words (Word count: Abstract - 181; Background 463; Materials and Methods - 628; Results - 1090; Discussion - 728; Conclusion - 83; References - 706; Figure legend - 587)

Keywords: Parkinson's disease; AMPK; mitochondria 


\section{Short Report}

\section{Abstract}

Emerging studies implicate energy dysregulation as an underlying trigger for

47 Parkinson's disease (PD), suggesting that a better understanding of the molecular pathways

48 governing energy homeostasis could help elucidate therapeutic targets for the disease. A

49 critical cellular energy regulator is AMP kinase (AMPK), which we have previously shown to

50 be protective in PD. However, precisely how AMPK function impacts on dopaminergic

51 neuronal survival and disease pathogenesis remains elusive. Here, we created a tissue-specific

52 AMPK-knockout mouse model where the catalytic subunits of AMPK are ablated in nigral

53 dopaminergic neurons. Using this model, we demonstrated that loss of AMPK function

54 promotes dopaminergic neurodegeneration and associated locomotor aberrations.

55 Accompanying this is a substantial reduction in the number of mitochondria in the surviving

56 AMPK-deficient nigral dopaminergic neurons, suggesting that an impairment in mitochondrial

57 biogenesis may underlie the observed PD-associated phenotypes. Importantly, the loss of

58 AMPK function enhances the susceptibility of nigral dopaminergic neurons in these mice to 6-

59 hydroxydopamine-induced toxicity. Taken together, these findings highlight the importance of

60 neuronal energy homeostasis by AMPK in PD and position AMPK pathway as an attractive

61 target for future therapeutic exploitation.

62

63

64

65

66

67

68

69 


\section{Short Report}

\section{Background}

Parkinson's disease (PD) is a prevalent neurodegenerative movement disorder that is characterized pathologically principally by the loss of dopaminergic (DA) neurons in the substantia nigra pars compacta (SNpc), which normally innervate the striatum to form the nigrostriatal pathway that is important for movement coordination. Although the mechanism underlying the pathology in PD remains unclear, emerging evidence suggests that energy dysregulation may predispose DA neurons toward degeneration. Notably, SNpc DA neurons are known to exhibit high energy demand as a result of their unique characteristics, including possessing a complex neuronal architecture, poorly myelinated axons and pacemaker firing driven by bioenergetically-expensive L-type $\mathrm{Ca}^{2+}$ currents (1-3). Given this, one could appreciate the need for SNpc DA neurons to maintain exquisite energy homeostasis for optimal function and survival.

A critical energy regulator in eukaryotic cells is $5^{\prime}$ adenosine monophosphate-activated protein kinase (AMPK). AMPK exists as a heterotrimer comprised of a catalytic $\alpha$ subunit ( $\alpha 1$ or $\alpha 2)$ and two regulatory subunits: $\beta$ ( $\beta 1$ or $\beta 2)$ and $\gamma$ subunits $(\gamma 1, \gamma 2$ or $\gamma 3)$. The enzyme becomes activated upon phosphorylation of the $\alpha$ subunit on threonine-172 in response to falling energy supply, e.g. ATP depletion or glucose starvation. Using phosphorylated AMPK as a surrogate marker for energy demand, we have recently reported that its physiological level is disproportionately elevated in the ventral midbrain (containing $\mathrm{SNpc}$ ) compared to various other regions in the mouse brain, which reflects its unique energy requirements (4). Conversely, AMPK activation is selectively disrupted in the midbrain of Parkin- and PTEN-induced kinase 1 (PINK1)-deficient mice; the loss of function of which in humans causes recessive Parkinsonism (4). Our results suggest that disruption of the AMPK pathway may precipitate PD whereas augmenting AMPK activity may mitigate the disease. Supporting this, we have demonstrated that AMPK activation ameliorates the disease phenotypes in Drosophila genetic 


\section{Short Report}

95 models of PD (5), and in a manner that is dependent on its downstream target peroxisome 96 proliferator-activated receptor gamma coactivator 1-alpha (PGC-1 $\alpha$ ), a regulator of

97 mitochondrial biogenesis (6). We further shown that pharmacological administration of AMPK 98 activators restores the levels of midbrain pAMPK and PGC-1 $\alpha$ expression in Parkin-deficient 99 mice (4).

100 To test our hypothesis that deficiency in AMPK activity predisposes DA neurons 101 toward degeneration, we report here the generation and characterization of a AMPK mutant 102 mouse model where the catalytic $\alpha 1$ and $\alpha 2$ subunits are conditionally ablated in DA neurons. 103 The conditional AMPK knockout (AMPK-cKO) animal exhibits DA neuronal loss and 104 associated locomotor deficits. We also recorded a significant reduction in the number of mitochondria in AMPK-deficient neurons, that is consistent with the role of AMPK in

106 promoting mitochondrial biogenesis. Importantly, these mutant mice show enhanced 107 susceptibility to 6-hydroxydopamine (6-OHDA)-induced DA neurotoxicity. Taken together, 108 our findings highlight the importance of AMPK in DA neuronal homeostasis and its utility as 109 a potential therapeutic target for PD. 


\section{Short Report}

120

121

122

123

124 separated on a $1 \%$ agarose gel for visualization.

\section{Materials and Methods}

Antibodies. The following antibodies were used: monoclonal anti-phospho-AMPK $\alpha 1 / \alpha 2$

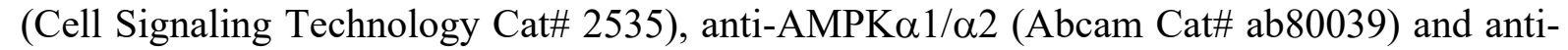
$\beta$-actin (Sigma Cat\# A5441); polyclonal anti-tyrosine hydroxylase (TH) (Pel-Freez Biologicals Cat\# P40101-0) and anti-TOM-20 (Santa Cruz Biotechnology Cat\# sc-11415); anti-mouse horseradish peroxidase and anti-rabbit horseradish peroxidise (GE Healthcare, UK).

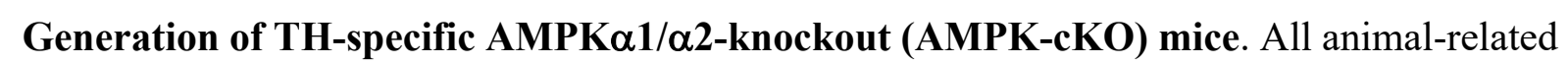
studies were approved by and conformed to the guidelines of the Institutional Animal Care and Use Committee (TTSH-NNI: TNI-15-13-021). Mice were maintained on a 12-h light/dark cycle with food and water available ad libitum. Transgenic TH-Cre mice (TH-Cre) were crossed with AMPK floxed mice that were homozygous for Prkaal (AMPKa1) and Prkaa2 (AMPK $\alpha 2)$ floxed genes $\left(A m p k^{f / f}\right)$, generating offspring $(50 \%)$ that were heterozygous for AMPK $\alpha 1 / \alpha 2$ floxed gene and TH-Cre (TH-Cre; Ampk $\left.k^{f /+}\right)$. TH-Cre; Ampk $k^{f /+}$ mice were backcrossed to parental $\mathrm{Ampk}^{f / f}$ mice to give TH-Cre; $\mathrm{Ampk}$ fff mice (12.5\%, AMPK-cKO mice) and $A m p k^{f f f}$ mice (12.5\%, AMPK-WT mice). Subsequently, AMPK-cKO mice were crossed with AMPK-WT mice to generate offspring of the same genotypes in the ratio of 1:1 based on Mendelian inheritance. Genomic DNA was extracted from mouse tails and the presence of THCre gene, AMPK $\alpha 1$ and AMPK $\alpha 2$ floxed genes were verified using PCR. A summary of the primers used and expected PCR products is provided in Table 1. The PCR products were 
Table 1. Primers used to genotype AMPK-WT and AMPK-cKO mice and their expected PCR products

149 Mice behavioral assessment. All animals were transported to the animal behavioral room for habituation to the novel room at least 30 minutes prior to experiments. Locomotor activity

151 recordings were carried out in an opaque open field arena $(45 \mathrm{x} 45 \mathrm{~cm})$. The testing apparatus

152 was enclosed in a ventilated, quiet and dim procedure room to minimize animals' stress levels

153 while allowing normal exploratory behavior. Animals were introduced into the arena and 154 allowed to explore freely for 10 minutes. Exploratory behavior was recorded using a 155 monochrome industrial camera (DMK 22AUC03, The Imaging Source, Taiwan) and

156 locomotor activity data was collected using the ANY-maze video tracking system. Motor 157 performance assays were also carried out using a rotarod as previously described (7). 


\section{Short Report}

Generation and characterization of 6-OHDA lesion PD model and apomorphine-induced

rotation assay. 7 AMPK-WT or 13 AMPK-cKO mice were lesioned with $1.5 \mu 1$ of $5 \mu \mathrm{g} / \mu \mathrm{L}$

161 6-hydroxydopamine (6-OHDA) using a method previously described (7). For every week post-

162 surgery, up to five weeks, the apomorphine-induced rotation assay was carried out and the number of rotations made in one minute was counted and recorded. 4 to 5-month-old wild-type C57B6 male mice injected with 6-OHDA were sacrificed via cervical dislocation. Mice brains were harvested and processed for western blot analysis as previously described (4). analysis. Mice brains were processed for immunohistochemistry and stereological analysis as previously described (7). For mitochondrial analysis, mice brains were sectioned in the coronal plane at $10 \mu \mathrm{m}$ thickness. Brain sections containing the SNpc were washed with washing buffer (0.1\% Triton X-100/PBS) before they were blocked with blocking buffer (5\% NGS in $0.1 \%$ Triton X-100/PBS) for one hour at room temperature. Brain sections were then incubated in anti-TH and anti-TOM20 antibodies in blocking buffer overnight at $4{ }^{\circ} \mathrm{C}$ and washed with washing buffer the next day. Following which, brains sections were incubated in secondary fluorescent antibodies at room temperature for four hours and washed with washing buffer

176 before being mounted on glass slides with Vectorshield mounting media containing DAPI.

177 Slides were viewed under the Olympus Fluoview FV1000 Laser Scanning Confocal 178 Microscope. Confocal images of 20 stained mice brain sections ( $1000 \mathrm{TH}-$ positive cells $)$ per group were analysed using a MATLAB algorithm that was modified from (8). 


\section{Short Report}

181 Statistical analysis. Statistical analyses were performed using the student's two-tailed

182 unpaired t-test $(* p<0.05, * * p<0.001$ or unless otherwise stated). All data was expressed as

183 mean (S.D.) generated from at least three independent experiments unless otherwise stated.

184

185

186

187

188

189

190

191

192

193

194

195

196

197

198

199

200

201

202

203

204 


\section{Short Report}

\section{Results}

206

207

208

209

210

211

212

213

214

215

216

217

218

219

220

221

222

223

224

225

226

227

\section{AMPK $\alpha$ expression is abolished in TH-expressing cells of AMPK-cKO mice brains}

We used the Cre/loxP-mediated recombination strategy to conditionally target the Prkaal/2 loci in the mouse brain. AMPK floxed mice $($ Prkaal/2fff $)$ in which the sequence encoding the catalytic site of both of the $\alpha$ subunits was flanked by loxP sequences has previously been described (9). To obtain DA neuronal-specific disruption of AMPK activity, Prkaal $/ 2^{f f f}$ mice were crossed with $\mathrm{TH}$-Cre transgenic mice expressing Cre recombinase under the control of the tyrosine hydroxylase promoter, to create TH-Cre; Prkaal/2fff $($ Prkaal/2ff$T H-c K O$ ) mice (herein refers to as AMPK cKO mice). Using PCR, we verified the presence of Prkaal/2fff in AMPK-WT mice and presence of both TH-Cre and Prkaal/2fff in AMPK-cKO mice (Fig. 1A). We further ascertained the deletion of AMPK $\alpha$ subunits in TH-expressing cells via immunofluorescence staining of AMPK-WT and AMPK-cKO mice brain sections using antibodies specific to AMPK- $\alpha 1$ and AMPK- $\alpha 2$ subunits. In both the nigral and striatal regions of AMPK-cKO mice, the expression of AMPK- $\alpha 1$ is significantly reduced $(p=0.0000222)$ (Fig. 1B-C and E). Unlike the predominantly cytoplasmic AMPK- $\alpha 1$ species, AMPK- $\alpha 2$ is normally localized to the nucleus (10). Thus, we examined AMPK- $\alpha 2$ expression mainly in the SNpc DA neurons where the cell bodies reside. We similarly recorded a significant reduction in AMPK- $\alpha 2$ expression in AMPK-cKO compared to AMPK-WT mice ( $p=0.00161)$ (Fig. 1D-E). Whereas dual knockout of the genes encoding AMPK- $\alpha 1$ and AMPK- $\alpha 2$ in the germline is embryonic lethal (11), AMPK-cKO mice do not exhibit developmental defects, gross abnormalities, or accelerated mortality (not shown). 


\section{Short Report}

228

229

230

231

232

233

234

235

236

237

238

239

240

241

242

243

244

245

246

247

248

249

250

251

252

\section{AMPK-cKO mice have reduced dopaminergic neuronal count and exhibit locomotor}

deficits compared to age-matched AMPK-WT mice

Working on the premise that AMPK is critical for DA neuronal survival, we anticipate that AMPK deficiency predisposes DA neurons toward degeneration. As early as threemonths-old, AMPK-cKO mice exhibit reduced TH-positive staining of the neurites in the striatum and the cell bodies in the SNpc (Fig. 2A). This difference is significant when quantified using an unbiased stereology-based approach $(p=0.00369)$ and persists even after aging to 22 months old ( $p=0.00641)$ (Fig. $2 \mathrm{~B})$. As SNpc DA neurons are predominantly responsible for modulating locomotion, we anticipate that the loss of these neurons in the AMPK-cKO mice will result in locomotor deficits, which are characteristic of mice with PD (12). The locomotor function of the AMPK-cKO mice and their wild-type counterparts were assessed using the open field and rotarod tests at various experimental time points $(3,8,12$ and 22 months old) in order to account for age-associated changes. In general, AMPK-cKO mice travel much less than AMPK-WT mice in the open field, as evident from the representative track plots (Fig. 2C). At three months old, the total distance travelled horizontally by the AMPK-cKO mice within the arena is significantly reduced when compared to age-matched AMPK-WT mice ( $p=0.00129)$ (Fig. 2C). Likewise, vertical rearing activity in the arena is also markedly reduced in the AMPK-cKO mice when compared to AMPK-WT mice and this difference persists through age ( $p=0.0253$ at 3 months; $p=0.00108$ at 8 months; $p=0.0101$ at 12 months; $p=0.0229$ at 22 months) (Fig. 2D). This is further supported by observations from the rotarod assay, a commonly used behavioural assessment for motor coordination, where AMPK-cKO mice falls off the accelerating rod more readily than AMPK-WT mice from eight to 22 months old ( $p=0.0000422$ at 8 months; $p=0.0120$ at 12 months; $p=0.0408$ at 22 months) (Fig. 2E), suggesting that motor coordination is impaired in the AMPK-cKO mice, especially as they age. Taken together, these findings indicate that loss of AMPK function predisposes 


\section{Short Report}

253 SNpc DA neurons to earlier degeneration, which manifests phenotypically in the form of

254 locomotor deficits.

\section{AMPK-cKO mice display aberrations in mitochondrial homeostasis}

To better understand the molecular underpinnings of our observations, we examined for any changes in terms of mitochondrial homeostasis, considering that the protective effects of AMPK in ameliorating PD-related phenotypes is mediated by its downstream effector and mitochondrial biogenesis regulator PGC-1 $\alpha$ (6). From the representative confocal images, there are fewer mitochondria (reduction in TOM20 staining) in the TH-expressing nigral DA neurons of AMPK-cKO mice compared to AMPK-WT mice (Fig. 3A). When quantified using a MATLAB algorithm, this reduction in the number of mitochondria is significant ( $p=0.0169$ ) (Fig. 3B), indicating the possibility of impaired mitochondrial biogenesis or enhanced mitophagy (clearance of damaged mitochondria). Since mitophagy is typically preceded by mitochondrial fission, we examined whether the mitochondria in AMPK-deficient nigral DA neurons tended toward fragmentation. However, the average mitochondrial sizes and aspect ratios were comparable between the AMPK-WT and AMPK-cKO groups ( $p=0.174)$ (Fig. 3B), suggesting that impaired mitophagy is an unlikely explanation for the reduction in the number of mitochondria in AMPK-deficient nigral DA neurons. Collectively, these findings indicate that AMPK deficiency affects mitochondrial homeostasis, in particular mitochondrial

272 biogenesis. 


\section{Short Report}

AMPK-cKO mice are more susceptible to 6-hydroxydopamine (6-OHDA)-mediated toxicity

Next, we wondered if these mice would be more susceptible to mitochondrial toxins given the changes in mitochondrial homeostasis. To study this, we subjected them to a PDrelated toxin 6-hydroxydopamine (6-OHDA) that is known to form free radicals and potently inhibit mitochondrial complexes I and IV. In wild-type mice injected with 6-OHDA, levels of the phosphorylated and active form of AMPK (pAMPK) is upregulated in the ventral midbrain region (containing SNpc) of the injected hemisphere (Fig. 4A), possibly a result of mitochondrial dysfunction that triggers a consequent reduction in ATP production (13). With the knowledge that functional AMPK signalling is required for prompt cellular response to metabolic changes and stresses (14), we speculate that this upregulation of AMPK activity may mediate a protective response against 6-OHDA-mediated toxicity and accordingly, loss of AMPK function may enhance susceptibility towards this toxin. Indeed, there is a significant reduction in the number of TH-positive nigral DA neurons on the injected (ipsilateral) side of AMPK-cKO mice compared to that of AMPK-WT mice ( $p=0.0117)$ (Fig. 4 C). This can be visualized rather apparently from the representative images of mice brain sections stained with TH (Fig. 4D). Unlike the AMPK-WT mice, AMPK-cKO mice have complete lesioning of the striatum at five weeks post-lesion accompanying the dramatic reduction in SNpc TH-staining (Fig. 4C-D). Loss of DA neurons unilaterally gives rise to a rotational phenotype in the contralateral direction when these mice are injected with apomorphine, a DA agonist (15), which can be quantified to reflect the severity of the lesion. From two to five weeks postsurgery, AMPK-cKO mice exhibit more pronounced rotational behaviour compared to AMPKWT mice $(p=0.0225$ at week $2 ; p=0.00126$ at week $3 ; p=0.00621$ at week $4 ; p=0.0141$ at week 5) (Fig. 4B). Collectively, these results show that the ablation of AMPK catalytic subunits aggravates 6-OHDA-induced DA neuronal loss. 


\section{Short Report}

301

302

303

304

305

306

307

308

309

310

311

312

313

314

315

316

317

318

319

320

321

322

323

324

325

\section{Discussion}

To study the role of AMPK in DA neurons and PD pathogenesis, we generated a conditional AMPK-KO mouse model where the catalytic alpha subunits of AMPK are ablated in TH-expressing DA neurons using the Cre/loxP-mediated recombination strategy. AMPKcKO mice exhibit accelerated DA neuronal loss and associated locomotor deficits. Accompanying this is a reduction in the number of mitochondria in the surviving AMPKdeficient nigral DA neurons, suggesting that aberrant mitochondrial biogenesis may underlie the PD-associated phenotypes observed during AMPK deficiency. Importantly, the AMPKcKO mice are more susceptible to DA neurotoxicity mediated by a mitochondrial and PDrelated toxin, 6-OHDA. Taken together, our findings highlight the importance of AMPK in DA neuronal homeostasis and thereby its therapeutic potential for PD.

To date, studies that support a neuroprotective role of AMPK in PD mostly employed an overexpression or activation strategy, including our previous study in the Drosophila (5). To confirm these findings, it is necessary to evaluate the effects of AMPK ablation, which is anticipated to promote DA neurodegeneration. However, in vivo AMPK-KO models designed to study AMPK's function in the brain are few and far between, much less in neurodegenerative diseases like PD (Viollet et al., 2009). One such study by Bayliss et al. created a similar tissuespecific knockout model where AMPK $\beta 1$ and $\beta 2$ regulatory subunits (rather than the catalytic AMPK $\alpha$ subunits) are specifically deleted in dopamine transporter (DAT)-expressing DA neurons (16). Using this model, they demonstrated that the protective effects of calorierestriction against MPTP toxicity occurs through the ghrelin-AMPK partnership. However, the baseline phenotypes of these conditional AMPK-KO mice were not reported, and it remains unclear what the effects of dysfunctional AMPK on DA neurons are. Addressing this gap, we showed here that loss of AMPK function in mice promotes nigral DA neurodegeneration and gives rise to locomotor deficits. Supporting this, several studies in Drosophila showed that 


\section{Short Report}

326

327

328

329

330

genetic loss-of-function of AMPK regulatory subunits ( $\beta$ or $\gamma$ ), or the expression of a dominantnegative form of AMPK results in overt neurodegeneration, reduction in locomotor activity and overall survival (17-19). In mice, ablating the catalytic subunits of AMPK in photoreceptors leads to early retinal defects including axonal retraction and synaptic alterations that is normally associated with aging (20). Although these findings are not specific to the context of nigral DA neurons, they collectively highlight the importance of an intact AMPK heterotrimer in neuronal survival. Our study concurs with these by further demonstrating that AMPK's catalytic function is critical to nigral DA neuronal homeostasis.

At the molecular level, we showed that the PD-associated phenotypes in AMPK-cKO mice occur concurrently with a reduction in the number of mitochondria, suggesting that aberrant mitochondrial biogenesis likely underlies the degeneration of AMPK-deficient DA neurons. As the energy powerhouse, mitochondria play an important role in energy metabolism in neurons, which are especially dependent on oxidative phosphorylation. Our previous work demonstrated that AMPK-mediated rescue of mitochondrial abnormalities in PD mutant flies is dependent on the mitochondrial biogenesis regulator PGC-1 $\alpha$ (6). Conversely, when AMPK activity is blocked in rat cortical neurons, the regulation of downstream PGC-1 $\alpha-\mathrm{NRF}-1$ pathway is interrupted and neuronal activity becomes uncoupled from mitochondrial energy metabolism (21). In line with this, we found that the mitochondria numbers in nigral DA neurons of AMPK-deficient mice are reduced, reflecting a dysfunctional AMPK-PGC-1 $\alpha$ axis. Since the ability of the cell to ensure a steady supply of healthy mitochondria is impaired, mitochondrial reactive oxygen species (mROS) may be elevated, as reported in AMPK-KO mouse embryonic fibroblasts that undergo premature senescence due to elevated mROS (22). Given this, it seems logical that the AMPK-cKO mice are more vulnerable to 6-OHDA, whose toxicity is largely accepted to be mediated through oxidative stress (23). We (i.e. current observations) and others have shown that 6-OHDA enhances AMPK phosphorylation, perhaps 


\section{Short Report}

351 as an attempt to cope with 6-OHDA-mediated toxicity (13). When mROS increases, AMPK

352 activates downstream mitochondria-related pathways, including PGC-1 $\alpha$-dependent

353 mitochondrial biogenesis and antioxidant response (22). When this protective mechanism is

354 impeded in AMPK-cKO mice subjected to a PD toxin that increases ROS, the nigral DA

355 neurons become more susceptible to degeneration. A similar observation has been reported

356 with yet another mitochondrial and PD-related toxin MPTP, where pharmacological or genetic

357 inhibition of AMPK further reduces cell viability (24). Taken together, these findings

358 demonstrate that mitochondrial dyshomeostasis occurs during AMPK deficiency and this

359 compromises the ability of nigral DA neurons to elicit a protective response in face of

360 mitochondria-related stress.

\section{Conclusion}

In summary, our findings that AMPK deficiency in nigral DA neurons promotes their

364 degeneration and results in locomotor aberrations in mice, supporting the protective role of

365 AMPK in PD. We further demonstrated that changes in mitochondrial homeostasis are involved when AMPK activity is abolished in nigral DA neurons, and this correspondingly enhances vulnerability against the mitochondrial and PD toxin 6-OHDA. Our study highlights the importance of AMPK in DA neuronal homeostasis, implicating it as a potential molecular and therapeutic target for disease intervention. 


\section{Short Report}

376

377

378

379

380

381

382

383

384

385

386

387

388

389

390

391

392

Availability of supporting data

400 The datasets used and/or analysed during the current study are available from the 


\section{Short Report}

401

corresponding author on reasonable request.

402

403 Competing interests

404 The authors declare that they have no competing interests.

405

406 Funding

407 This work was supported by grants from the National Medical Research Council Large 408 Collaborative Grant - SPARK2 (LKL), IRNMRPD21AKL. HL was supported by a graduate 409 scholarship from the National University of Singapore Graduate School for Integrative 410 Sciences and Engineering.

411

412 Authors' contributions

413 HL, GG, WZ, CHY, JT performed the experiments and analysed the data. XS, LKP provided 414 critical reagents and intellectual contributions. HL, LKL conceptualized the study, interpreted 415 the results and wrote the manuscript. All authors read and approved the final manuscript.

416

417 Acknowledgements

418 We thank Dr. Willcyn Tang for the technical support rendered to the study.

419

420 Authors' information

$421 \quad$ Affiliations

422 Lee Kong Chian School of Medicine, Nanyang Technological University Singapore, 423 Singapore.

424 Liting Hang, Geraldine W.Y. Goh \& Kah-Leong Lim

425 NUS Graduate School for Integrative Sciences and Engineering, National University of 


\section{Short Report}

426 Singapore, Singapore.

427 Liting Hang

428 Department of Physiology, National University of Singapore, Singapore.

429 Liting Hang \& Shengli Xu

430 Department of Microbiology \& Immunology, National University of Singapore,

431 Singapore.

432 Kong Peng, Lam

433 Department of Research, National Neuroscience Institute, Singapore.

434 Liting Hang, Geraldine W.Y. Goh, Ziyin Wang, Huey Ching Choong \& John Thundyil

435 A*STAR Bioprocessing Technology Institute, Singapore.

436 Shengli Xu \& Kong Peng, Lam

437

438 Corresponding author

439 Correspondence to Kah-Leong Lim.

440

441

442

443

444

445

446

447

448

449

450 


\section{Short Report}

451

452

453

454

455

456

457

458

459

460

461

462

463

464

465

466

467

468

469

470

471

472

473

474

475

476

477

478

479

480

481

482

483

484

485

486

487

488

489

490

491

492

493

494

495

496

497

498

\section{References}

1. Braak H, Del Tredici K. Poor and protracted myelination as a contributory factor to neurodegenerative disorders. Neurobiology of aging. 2004;25(1):19-23.

2. Pacelli C, Giguere N, Bourque MJ, Levesque M, Slack RS, Trudeau LE. Elevated Mitochondrial Bioenergetics and Axonal Arborization Size Are Key Contributors to the Vulnerability of Dopamine Neurons. Current biology : CB. 2015;25(18):2349-60.

3. Surmeier DJ, Obeso JA, Halliday GM. Selective neuronal vulnerability in Parkinson disease. Nat Rev Neurosci. 2017;18(2):101-13.

4. Hang L, Thundyil J, Goh GWY, Lim KL. AMP Kinase Activation is Selectively Disrupted in the Ventral Midbrain of Mice Deficient in Parkin or PINK1 Expression. Neuromolecular medicine. 2019;21(1):25-32.

5. $\quad \mathrm{Ng} \mathrm{CH}$, Guan MS, Koh C, Ouyang X, Yu F, Tan EK, et al. AMP kinase activation mitigates dopaminergic dysfunction and mitochondrial abnormalities in Drosophila models of Parkinson's disease. The Journal of neuroscience : the official journal of the Society for Neuroscience. 2012;32(41):14311-7.

6. Ng CH, Basil AH, Hang L, Tan R, Goh KL, O'Neill S, et al. Genetic or pharmacological activation of the Drosophila PGC-1alpha ortholog spargel rescues the disease phenotypes of genetic models of Parkinson's disease. Neurobiology of aging. 2017;55:33-7.

7. Zhang C-W, Tai YK, Chai B-H, Chew KCM, Ang E-T, Tsang F, et al. Transgenic Mice Overexpressing the Divalent Metal Transporter 1 Exhibit Iron Accumulation and Enhanced Parkin Expression in the Brain. Neuromolecular medicine. 2017;19(2-3):375-86.

8. Poon VY, Goh C, Voorhoeve PM, Fivaz M. High-content imaging of presynaptic assembly. Front Cell Neurosci. 2014;8:66-.

9. Nakada D, Saunders TL, Morrison SJ. Lkb1 regulates cell cycle and energy metabolism in haematopoietic stem cells. Nature. 2010;468(7324):653-8.

10. Turnley AM, Stapleton D, Mann RJ, Witters LA, Kemp BE, Bartlett PF. Cellular distribution and developmental expression of AMP-activated protein kinase isoforms in mouse central nervous system. Journal of neurochemistry. 1999;72(4):1707-16.

11. Viollet B, Athea Y, Mounier R, Guigas B, Zarrinpashneh E, Horman S, et al. AMPK: Lessons from transgenic and knockout animals. Front Biosci (Landmark Ed). 2009;14:19-44. 12. Taylor TN, Greene JG, Miller GW. Behavioral phenotyping of mouse models of Parkinson's disease. Behavioural brain research. 2010;211(1):1-10.

13. Kim TW, Cho HM, Choi SY, Suguira Y, Hayasaka T, Setou M, et al. (ADP-ribose) polymerase 1 and AMP-activated protein kinase mediate progressive dopaminergic neuronal degeneration in a mouse model of Parkinson's disease. Cell death \& disease. 2013;4:e919.

14. Reznick RM, Zong H, Li J, Morino K, Moore IK, Yu HJ, et al. Aging-associated reductions in AMP-activated protein kinase activity and mitochondrial biogenesis. Cell Metab. 2007;5(2):151-6.

15. Hudson JL, van Horne CG, Stromberg I, Brock S, Clayton J, Masserano J, et al. Correlation of apomorphine- and amphetamine-induced turning with nigrostriatal dopamine content in unilateral 6-hydroxydopamine lesioned rats. Brain research. 1993;626(1-2):167-74. 16. Bayliss JA, Lemus MB, Stark R, Santos VV, Thompson A, Rees DJ, et al. GhrelinAMPK Signaling Mediates the Neuroprotective Effects of Calorie Restriction in Parkinson's Disease. 2016;36(10):3049-63.

17. Johnson EC, Kazgan N, Bretz CA, Forsberg LJ, Hector CE, Worthen RJ, et al. Altered metabolism and persistent starvation behaviors caused by reduced AMPK function in Drosophila. PloS one. 2010;5(9):e12799.

18. Spasic MR, Callaerts P, Norga KK. Drosophila alicorn is a neuronal maintenance factor 


\section{Short Report}

499

500

501

502

503

504

505

506

507

508

509

510

511

512

513

514

515

516

517

518

519

520

521

522

523

524

525

526

527

528

529

530

531

532

533

protecting against activity-induced retinal degeneration. The Journal of neuroscience : the official journal of the Society for Neuroscience. 2008;28(25):6419-29.

19. Tschäpe J-A, Hammerschmied C, Mühlig-Versen M, Athenstaedt K, Daum G, Kretzschmar D. The neurodegeneration mutant löchrig interferes with cholesterol homeostasis and Appl processing. EMBO J. 2002;21(23):6367-76.

20. Samuel MA, Voinescu PE, Lilley BN, de Cabo R, Foretz M, Viollet B, et al. LKB1 and AMPK regulate synaptic remodeling in old age. Nature neuroscience. 2014;17(9):1190-7.

21. Yu L, Yang SJ. AMP-activated protein kinase mediates activity-dependent regulation of peroxisome proliferator-activated receptor gamma coactivator-1alpha and nuclear respiratory factor 1 expression in rat visual cortical neurons. Neuroscience. 2010;169(1):2338.

22. Rabinovitch RC, Samborska B, Faubert B, Ma EH, Gravel SP, Andrzejewski S, et al. AMPK Maintains Cellular Metabolic Homeostasis through Regulation of Mitochondrial Reactive Oxygen Species. Cell Rep. 2017;21(1):1-9.

23. Choi WS, Yoon SY, Oh TH, Choi EJ, O'Malley KL, Oh YJ. Two distinct mechanisms are involved in 6-hydroxydopamine- and MPP+-induced dopaminergic neuronal cell death: role of caspases, ROS, and JNK. J Neurosci Res. 1999;57(1):86-94.

24. Choi JS, Park C, Jeong JW. AMP-activated protein kinase is activated in Parkinson's disease models mediated by 1-methyl-4-phenyl-1,2,3,6-tetrahydropyridine. Biochemical and biophysical research communications. 2010;391(1):147-51.

(1)

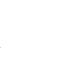




\section{Short Report}

\section{$534 \quad$ Figure legend}

Fig. 1. AMPK $\alpha$ expression is abolished in TH-positive cells of AMPK-cKO mice brains.

536 (A) Top, PCR results indicating the presence of $\mathrm{TH}$-Cre transgene (100bp product) in AMPK537 cKO mice and the internal positive control (324bp product) in AMPK-WT and AMPK-cKO 538 mice. Bottom, PCR results indicating the presence of Prkaal/2fff (450bp product) in both the AMPK-WT and AMPK-cKO mice. (B) Immunofluorescence labelling of striatal sections (Str) of AMPK-WT and AMPK-cKO mice brains with DAPI (blue, nucleus), TH (green) and AMPK $\alpha 1$ (red). Merged image shows colocalization of DAPI, TH and AMPK $\alpha 1$. (C) Same as (B) but in sections containing substantia nigra pars compacta (SNpc). (D) Same as (C) but red channel is AMPKa2. (E) Left, representative confocal images of the respective enlarged views from (C) and (D). Right, quantification of the percentage of TH-positive cells expressing AMPK $\alpha 1 / \alpha 2$. Statistical analysis: student's two-tailed unpaired t-test $\left({ }^{*} p<0.05\right.$ and ${ }^{* *} p<0.001$ compared to AMPK-WT mice).

Fig. 2. AMPK-cKO mice have reduced dopaminergic neuronal count and locomotor deficits compared to age-matched AMPK-WT mice. (A) Left, representative striatal sections and right, midbrain sections containing the SNpc of 3 months and 22 months old AMPK-WT and AMPK-cKO mice. The SNpc region is highlighted within the white dotted lines. Positive DAB staining of $\mathrm{TH}$ is indicated in brown. (B) Left, enlarged view of the SNpc regions highlighted within the white dotted lines from (A). Right, bar graph showing the stereological counts of nigral TH-positive DA neurons from 3 months and 22 months old AMPK-WT and AMPK-cKO mice. (C) Representative track plots, total distance travelled and (D) rearing activity of AMPK-WT ( $n=19, n=12, n=20, n=11)$ and AMPK-cKO mice $(n=19, n=12, n=25$,

$557 n=12)$ from the respective age groups $(3,8,12$ and 22 months old) during the open field test.

(E) AMPK-WT and AMPK-cKO mice latency to fall from the rotarod at the same age groups. 


\section{Short Report}

559 Statistical analysis: student's two-tailed unpaired t-test $\left({ }^{*} p<0.05\right.$ and ${ }^{*} p<0.001$ compared to

560 AMPK-WT mice at respective time points, ${ }^{\mathrm{a}} p<0.05$ and ${ }^{\text {aa }} p<0.001$ compared to previous time 561 point within the same group).

563 Fig. 3. AMPK-cKO mice display aberrations in mitochondrial homeostasis. (A) 564 Representative images showing immunofluorescence labelling of SNpc-containing sections of 565 AMPK-WT and AMPK-cKO mice brains with DAPI (blue, nucleus), TH (green) and TOM20 566 (red, mitochondria). TOM20 staining of the cell located in the white dotted lined box is shown 567 in enlarged view. Merged image shows colocalization of DAPI, TH and TOM20. Scale bar:

568 20um. (B) Left to right, bar graphs showing the number of mitochondria per cell, average area 569 of mitochondria and line graph showing the distribution of the mitochondria aspect ratios 570 (mitochondria length to width ratios) between nigral TH-positive DA neurons of AMPK-WT and AMPK-cKO mice. Statistical analysis: student's two-tailed unpaired t-test $\left({ }^{*} p<0.05\right.$ compared to AMPK-WT).

Fig. 4. AMPK-cKO mice are more susceptible to 6-hydroxydopamine (6-OHDA)mediated toxicity. (A) Representative immunoblots showing the expression of pAMPK, AMPK and actin across selected brain regions (cortex, striatum and ventral midbrain) of wildtype mice in the absence or presence of 6-OHDA. (B) Number of apomorphine-induced contralateral rotations of 6-OHDA-lesioned AMPK-WT mice $(n=7)$, 6-OHDA-lesioned AMPK-cKO mice $(n=13)$ and sham controls $(n=6)$ from week 1 to week 5 post-surgery. (C)

580 Stereological counts of TH-positive cells in the contralateral (non-lesioned) and ipsilateral 581 (lesioned) sides of brain sections containing SNpc from AMPK-WT or AMPK-cKO mice. (D)

582 Representative immunohistochemical images of the SNpc and striatal (Str) sections of AMPK-

583 WT or AMPK-cKO mice five weeks post-surgery, arranged from anterior to posterior position. 


\section{Short Report}

584 Positive brown stains indicate presence of TH-positive cells. Statistical analysis: student's two585 tailed unpaired t-test $\left({ }^{*} p<0.05\right.$ compared to AMPK-WT mice, ${ }^{*} p<0.05$ compared to 586 contralateral side). 
Figure 1

A

$$
\begin{aligned}
& \text { TH-Cre - }+ \\
& 500= \\
& 400= \\
& 300=- \\
& 200- \\
& 100- \\
& \text { WT cKO }
\end{aligned}
$$

AMPK f/f +/+ +/+

$$
\begin{aligned}
& 500=- \\
& 400= \\
& 300- \\
& 200- \\
& 100- \\
& \text { WT cKO }
\end{aligned}
$$

AMPK $\alpha 1$

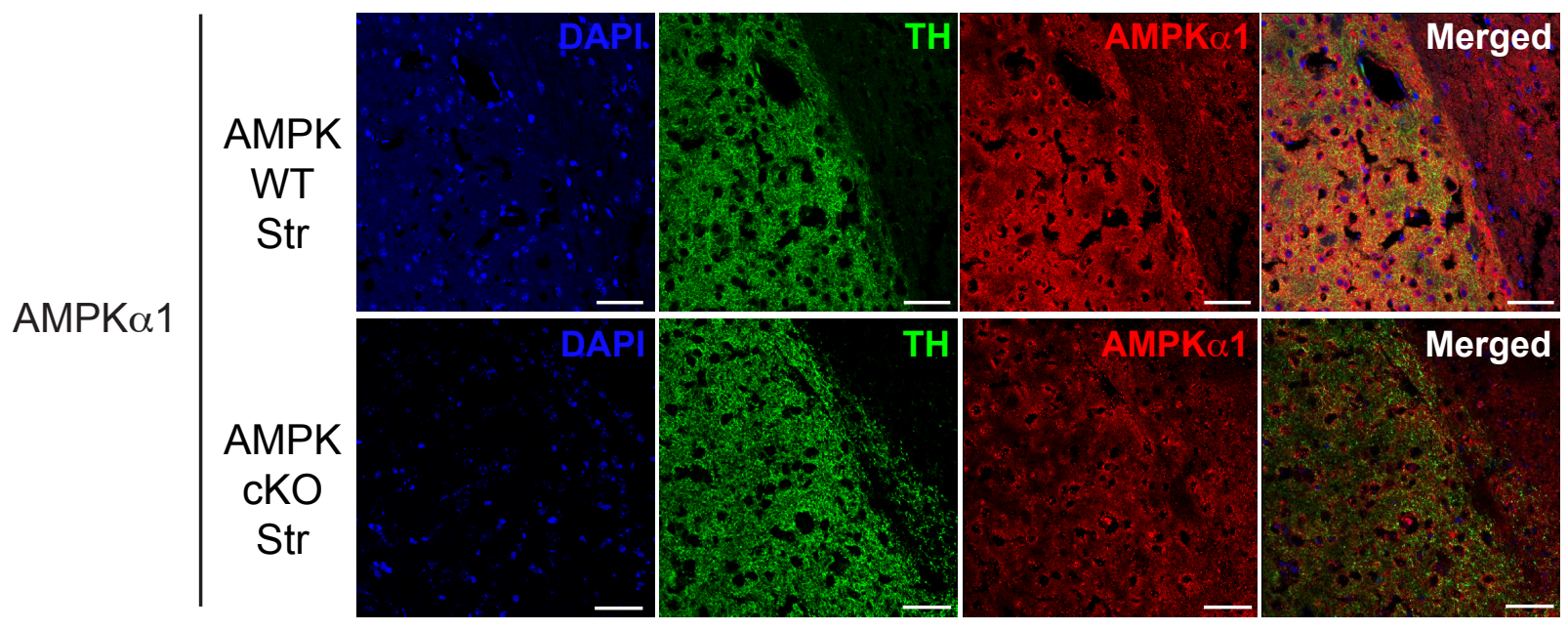

B
C

AMPK $\alpha 1$

$$
\begin{array}{|l|} 
\\
\text { AMPK } \\
\text { WT } \\
\text { SNpc } \\
\\
\\
\text { AMPK } \\
\text { CKO } \\
\text { SNpc }
\end{array}
$$

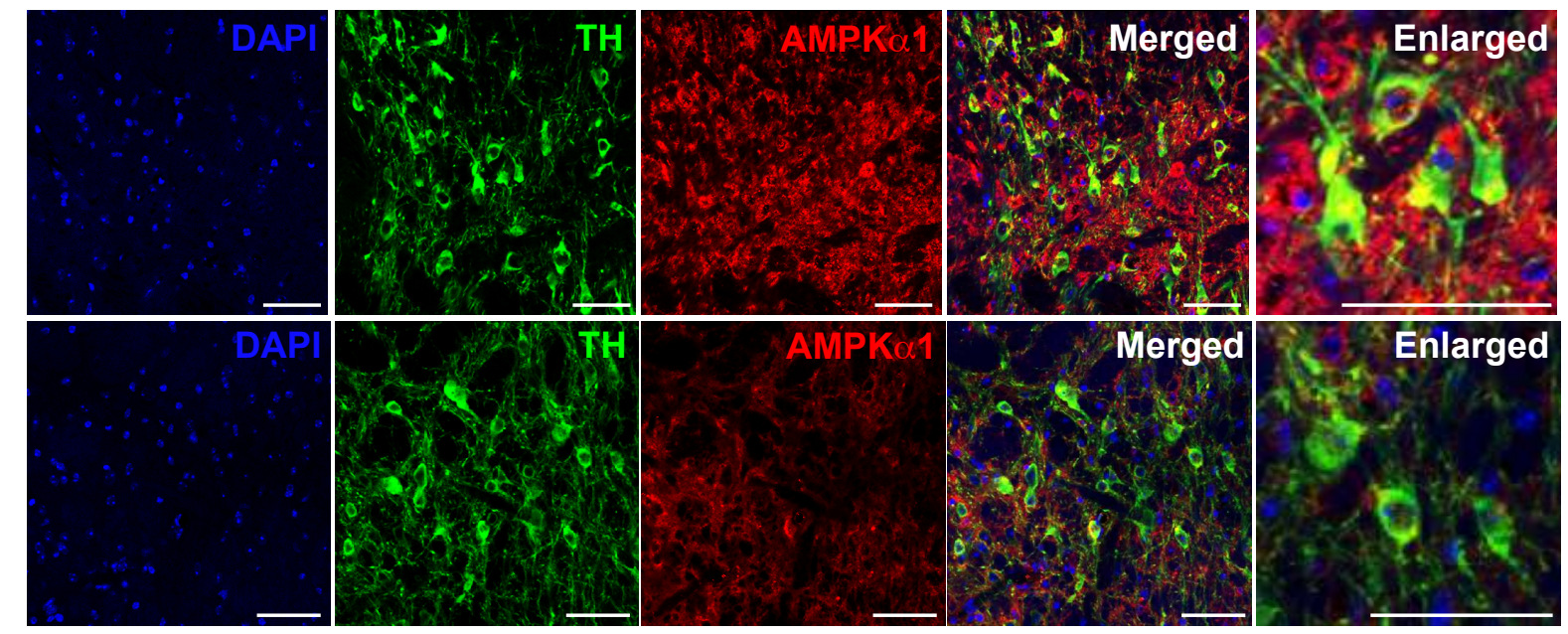

D

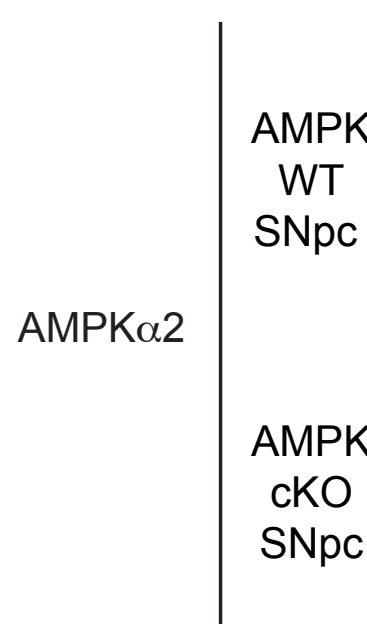

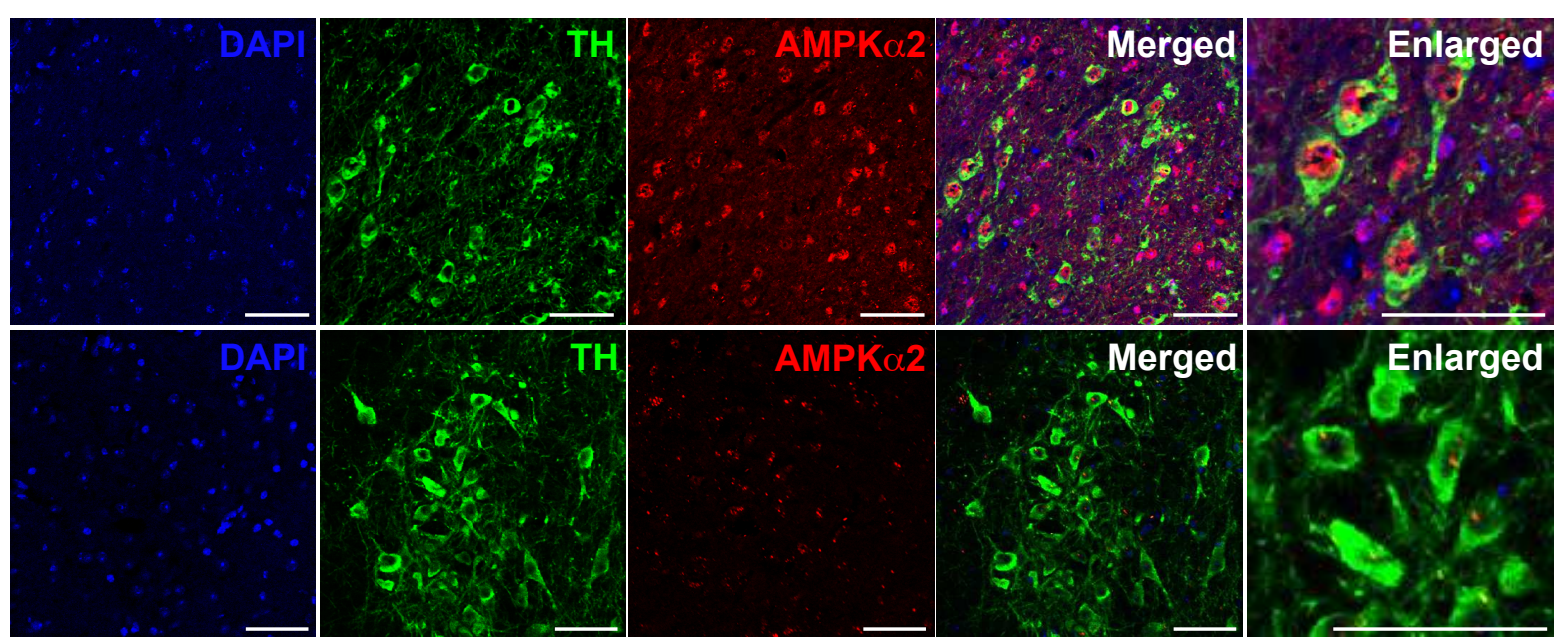

\section{AMPK-WT AMPK-cKO}

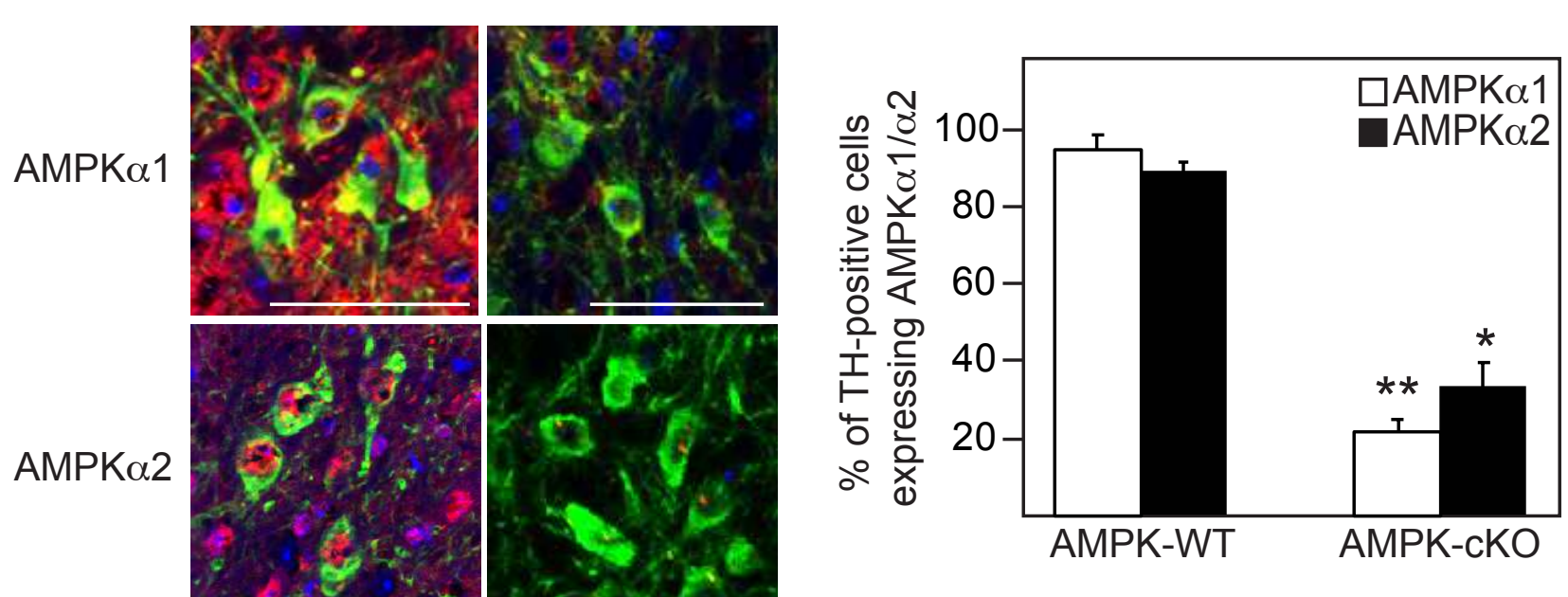


Figure 2

A

3 months 22 months

3 months

22 months

AMPK-WT

Str

AMPK-cKO

Str

B

3 months 22 months

AMPK-WT

$\mathrm{SNpc}$

AMPK-cKO

$\mathrm{SNpc}$

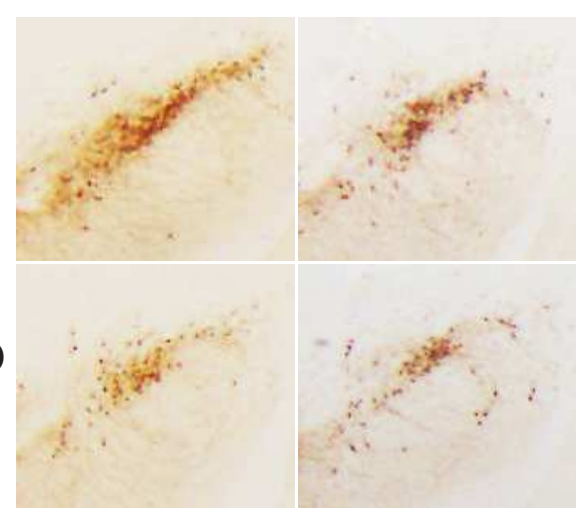

AMPK-WT
SNpc

AMPK-cKO $\mathrm{SNpc}$
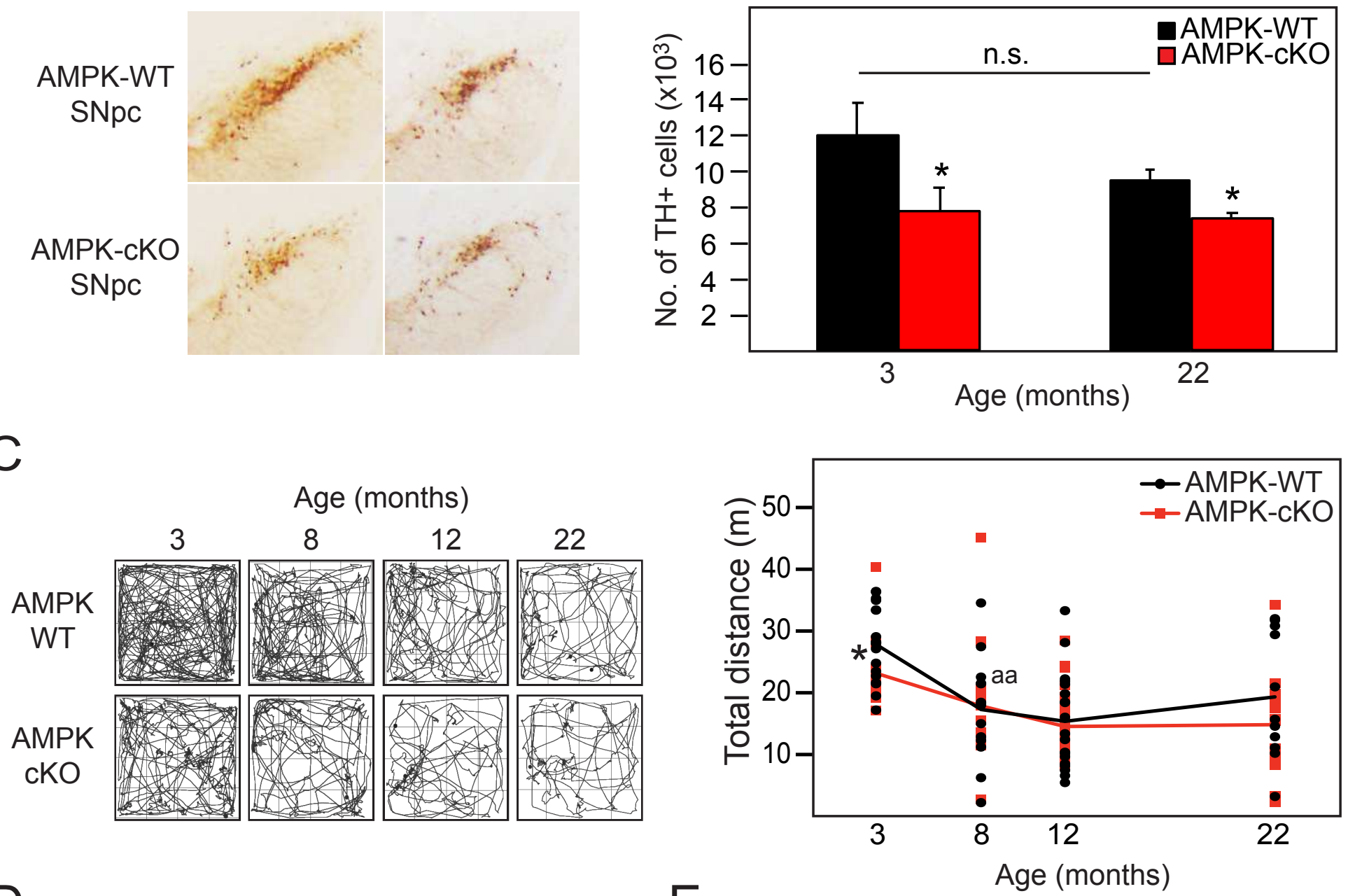

$\mathrm{D}$

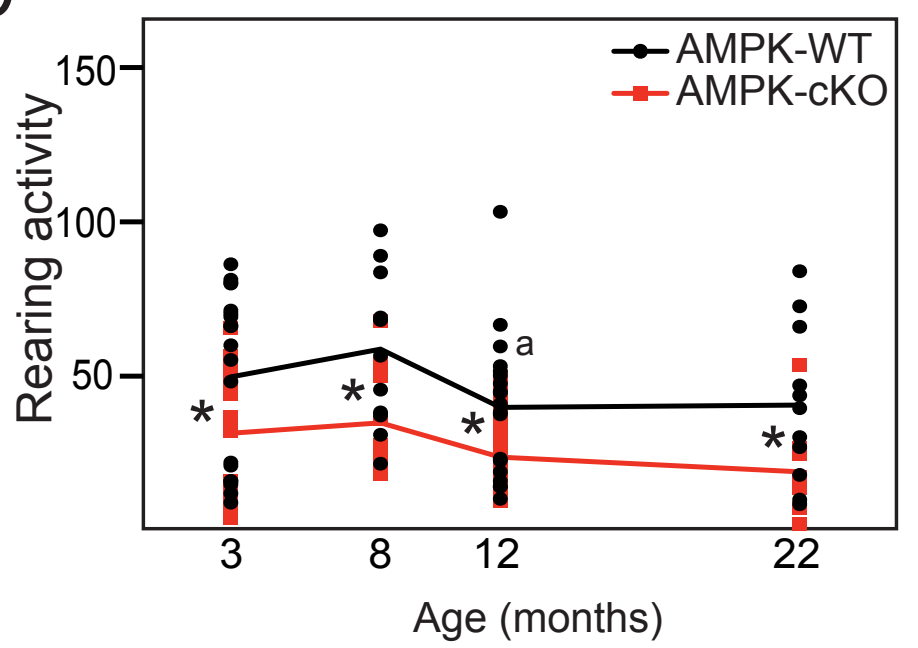

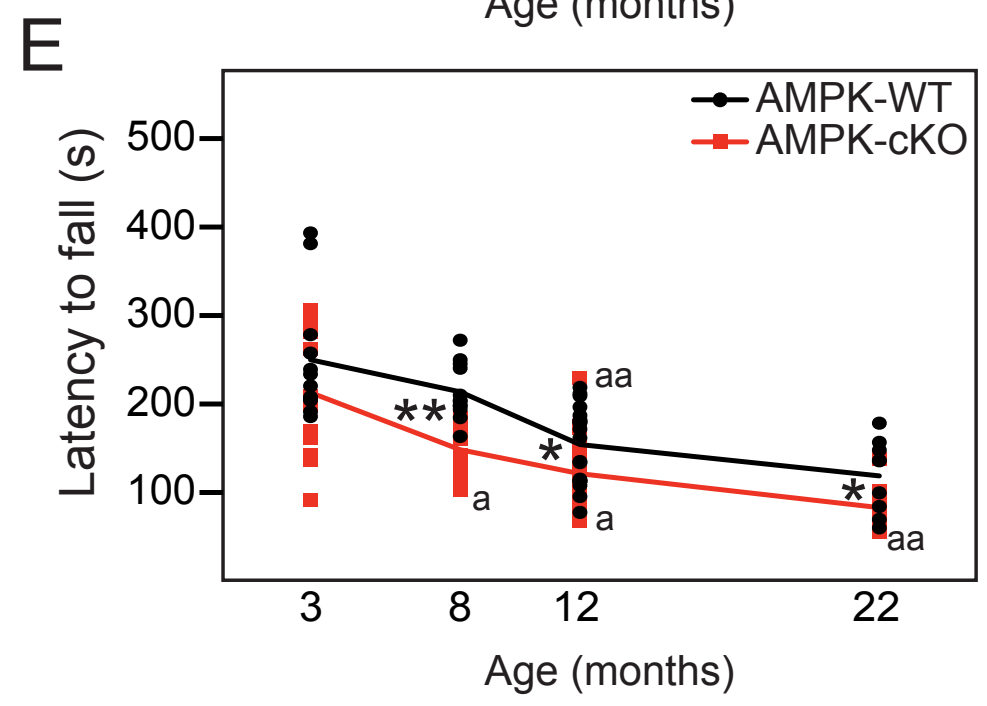


$A$

Figure 3

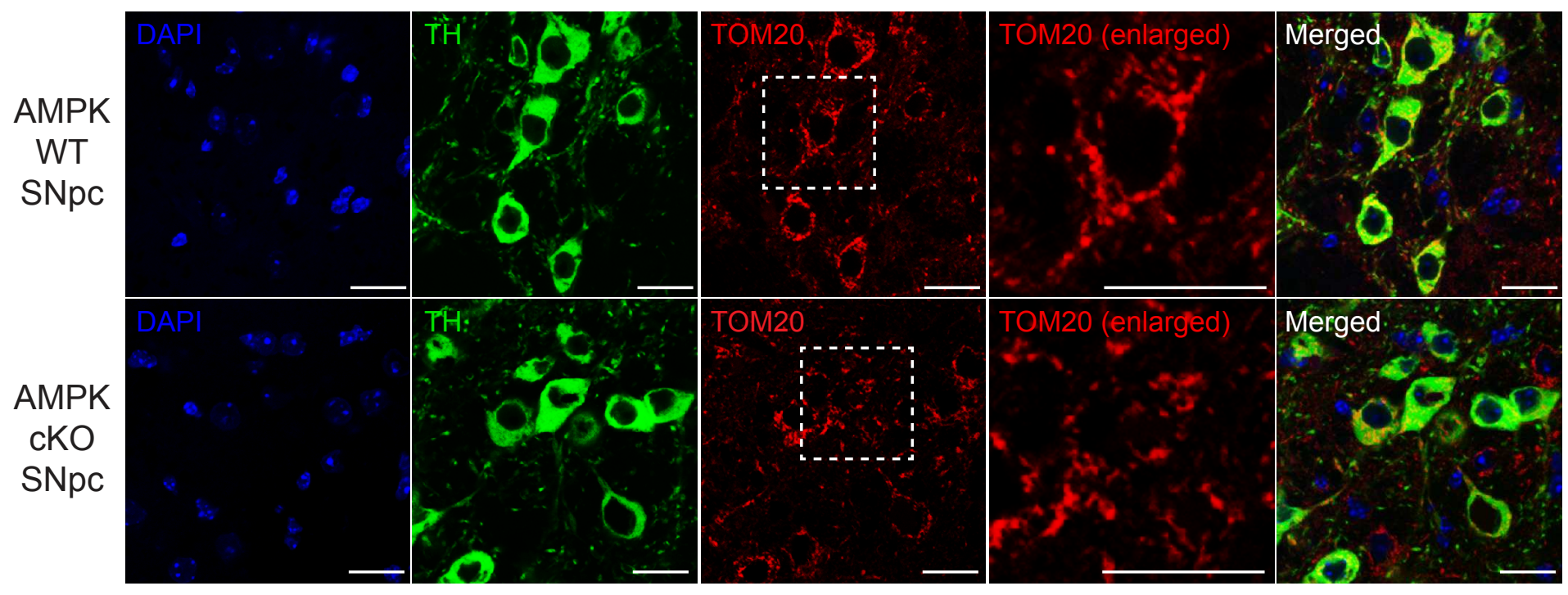

B
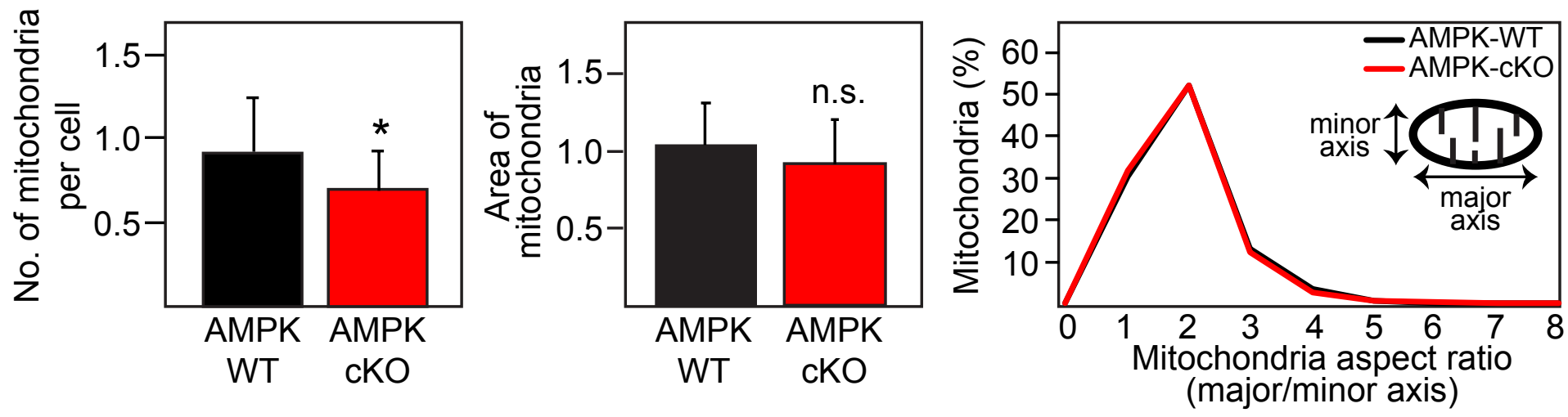
Figure 4

A

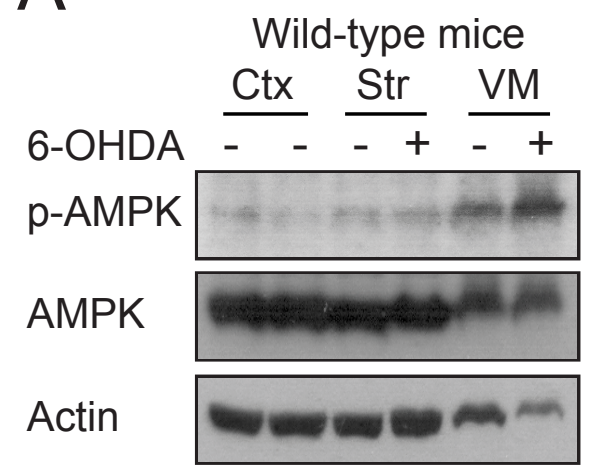

D

B

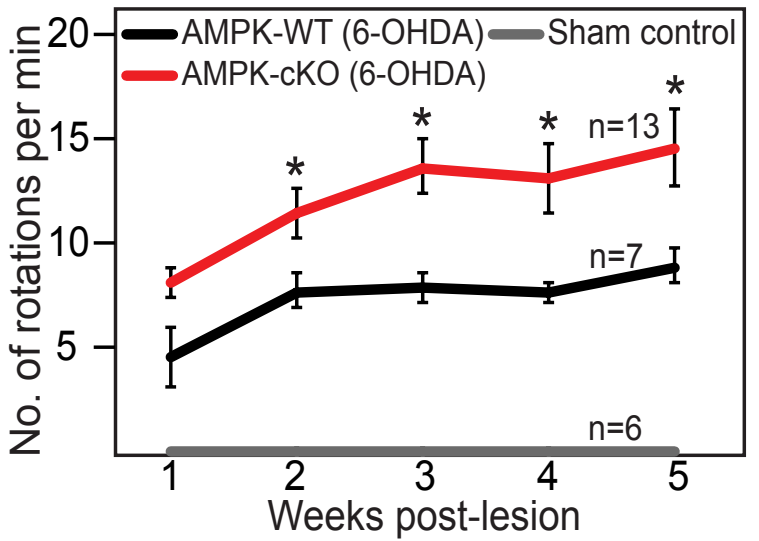

C

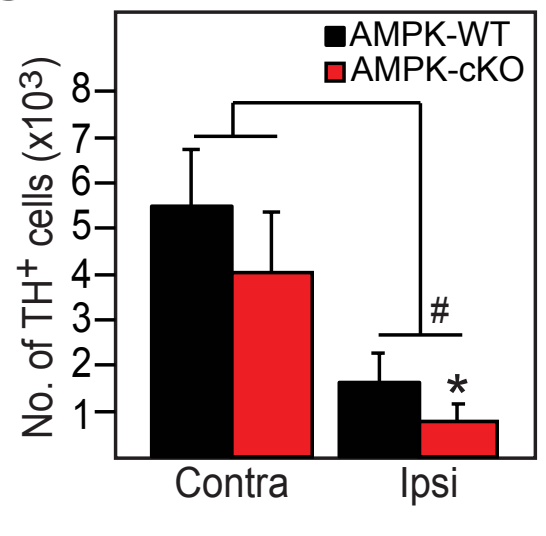

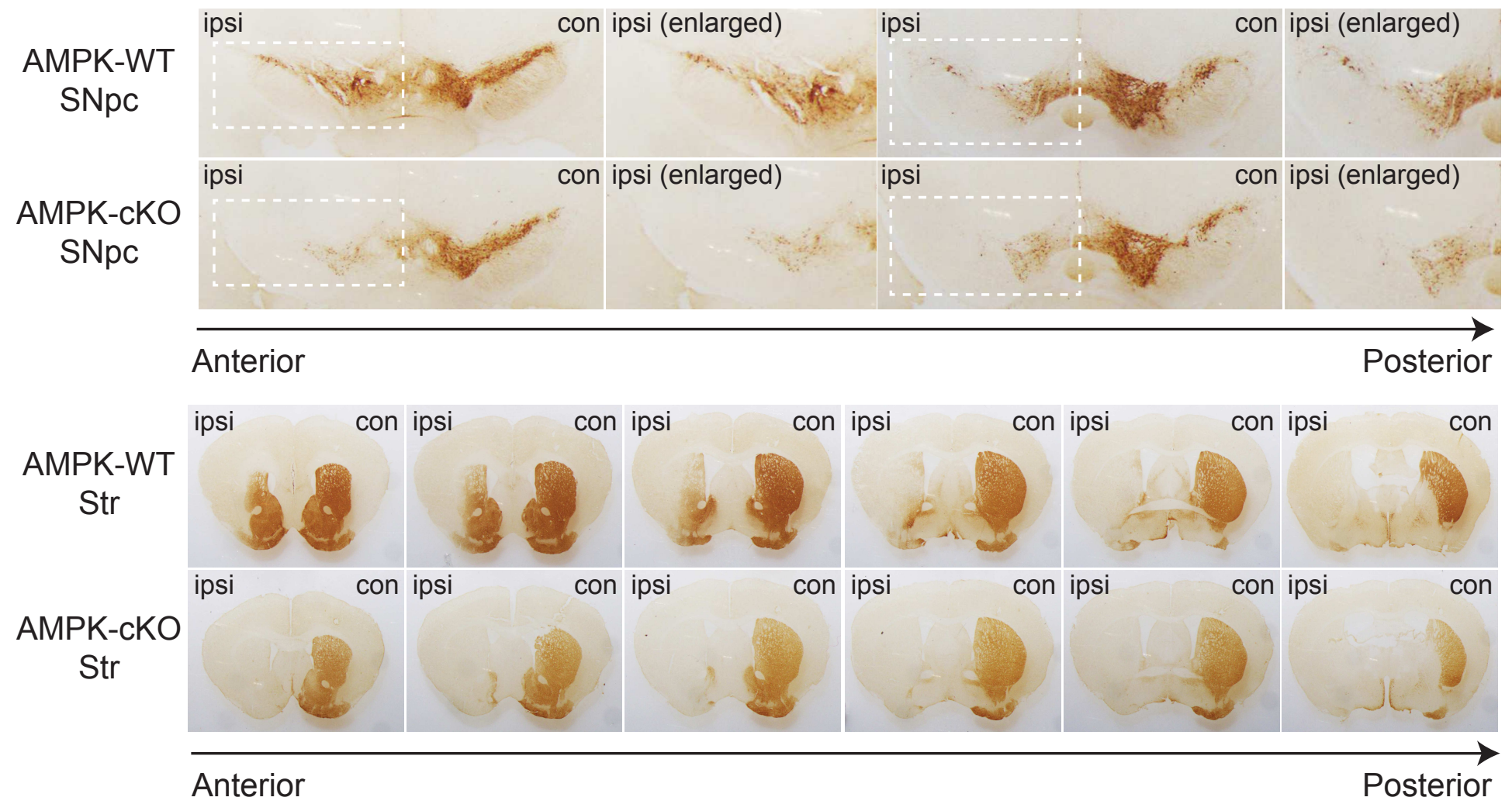




\section{Figures}

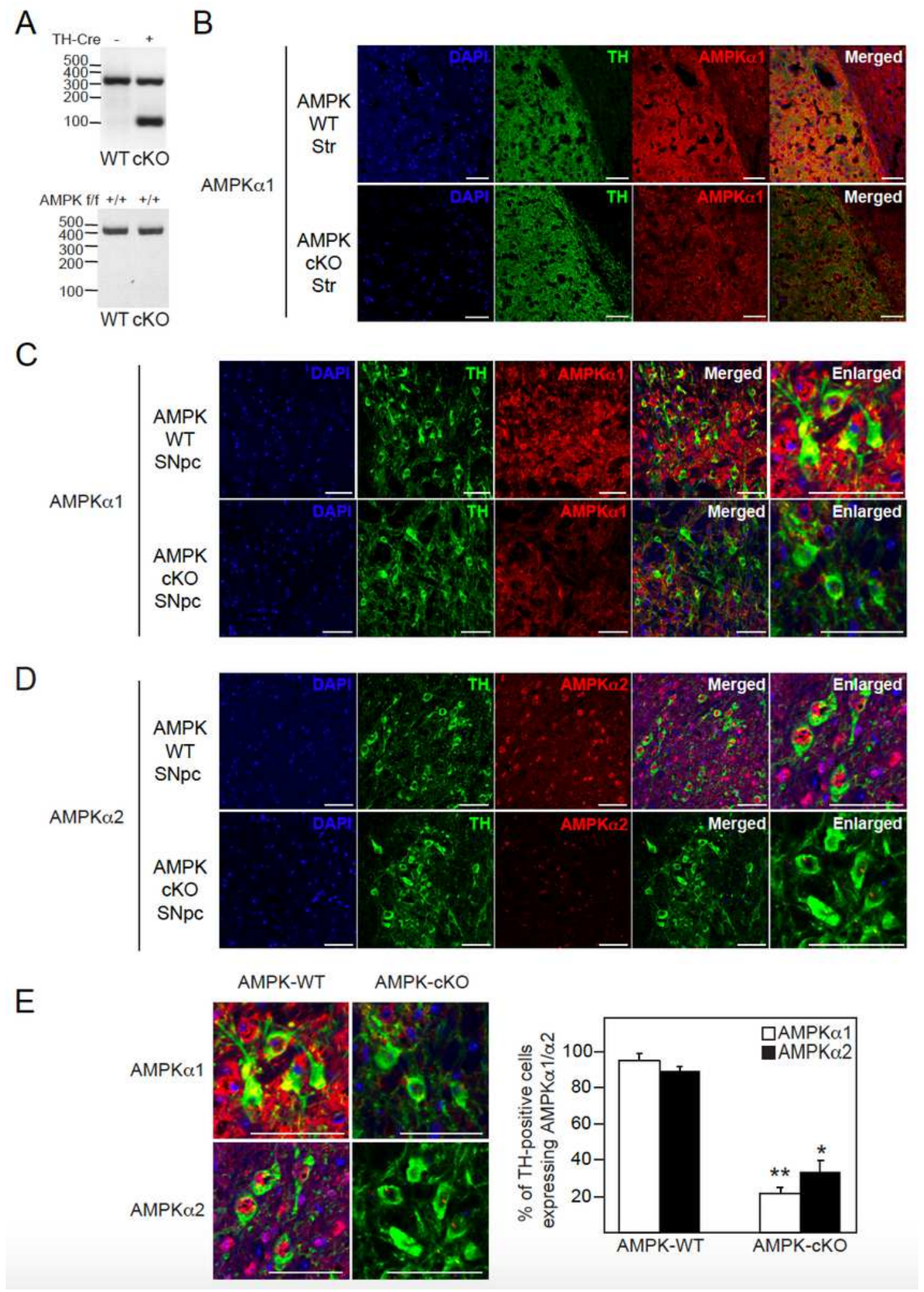

\section{Figure 1}

AMPKa expression is abolished in TH-positive cells of AMPK-cKO mice brains. (A) Top, PCR results indicating the presence of TH-Cre transgene (100bp product) in AMPKcKO mice and the internal positive control (324bp product) in AMPK-WT and AMPK-cKO mice. Bottom, PCR results indicating the presence 
of Prkaa1/2f/f (450bp product) in both the AMPK-WT and AMPK-cKO mice. (B) Immunofluorescence labelling of striatal sections (Str) of AMPK-WT and AMPK-cKO mice brains with DAPI (blue, nucleus), TH (green) and AMPKa1 (red). Merged image shows colocalization of DAPI, TH and AMPKa1. (C) Same as (B) but in sections containing substantia nigra pars compacta (SNpc). (D) Same as (C) but red channel is AMPKa2. (E) Left, representative confocal images of the respective enlarged views from (C) and (D). Right, quantification of the percentage of TH-positive cells expressing AMPKa1/a2. Statistical analysis: student's two-tailed unpaired t-test ( ${ }^{*} p<0.05$ and ${ }^{* *} p<0.001$ compared to AMPK-WT mice).

A
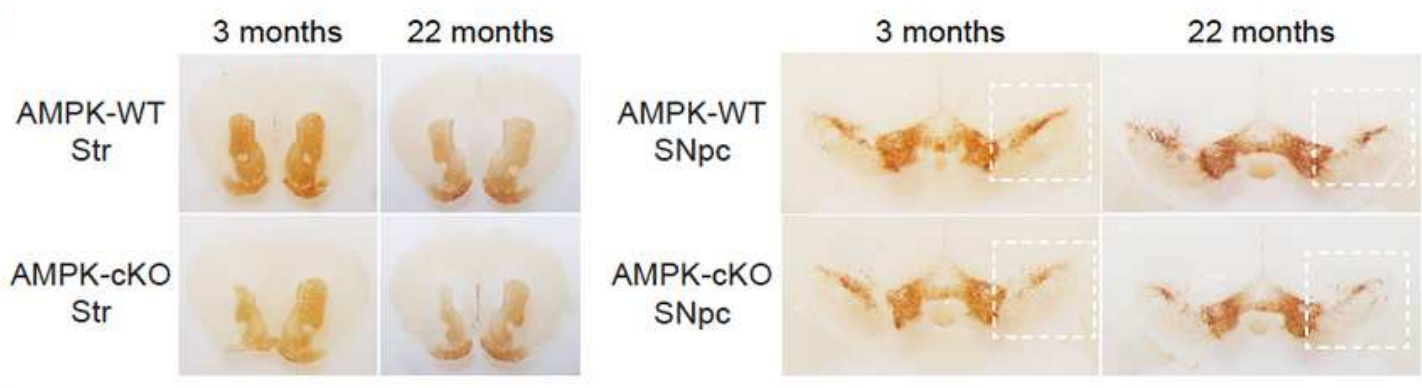

$\mathrm{B}$
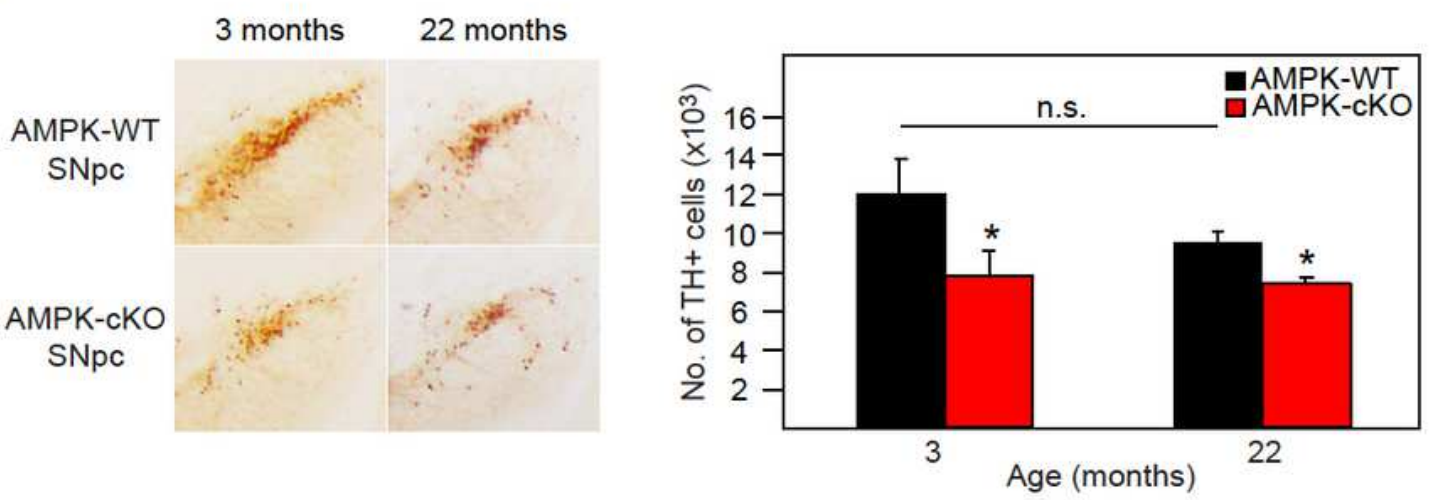

C

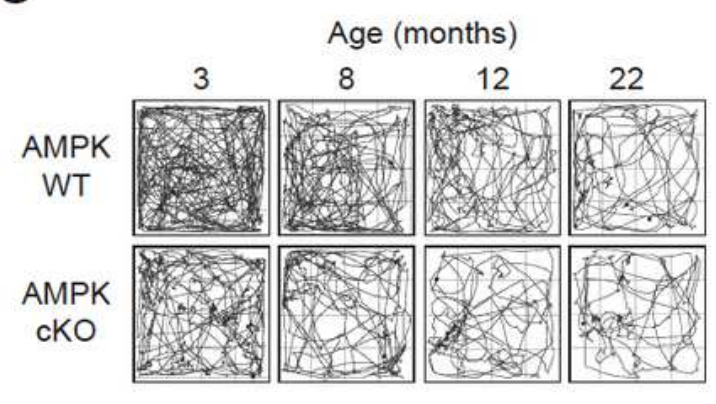

D

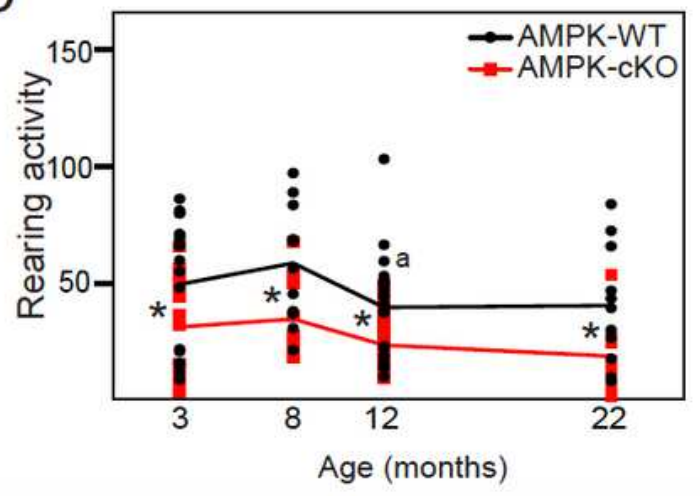

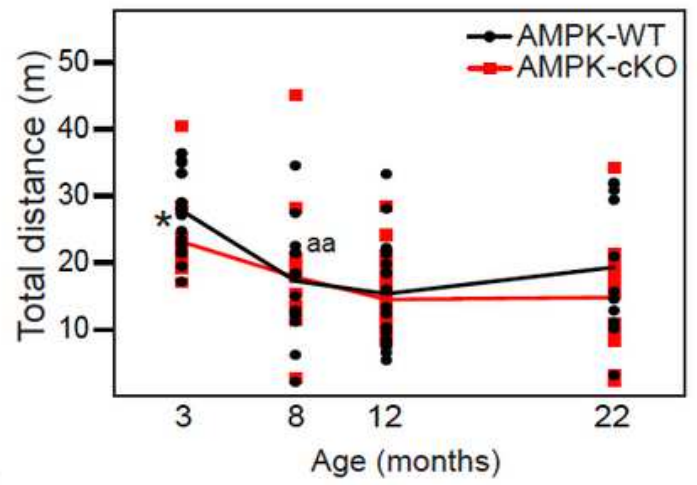

$E$

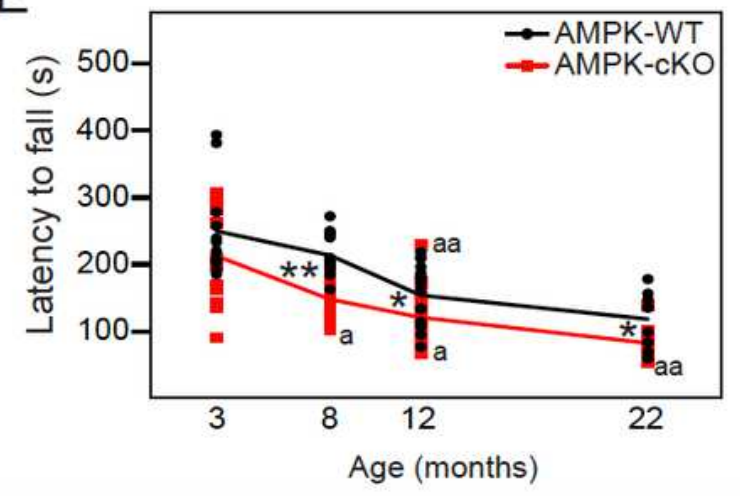




\section{Figure 2}

AMPK-cKO mice have reduced dopaminergic neuronal count and locomotor deficits compared to agematched AMPK-WT mice. (A) Left, representative striatal sections and right, midbrain sections containing the SNpc of 3 months and 22 months old AMPK-WT and AMPK-cKO mice. The SNpc region is highlighted within the white dotted lines. Positive DAB staining of TH is indicated in brown. (B) Left, enlarged view of the SNpc regions highlighted within the white dotted lines from (A). Right, bar graph showing the stereological counts of nigral TH-positive DA neurons from 3 months and 22 months old AMPK-WT and AMPK-cKO mice. (C) Representative track plots, total distance travelled and (D) rearing activity of AMPKWT $(n=19, n=12, n=20, n=11)$ and AMPK-cKO mice $(n=19, n=12, n=25, n=12)$ from the respective age groups $(3,8,12$ and 22 months old) during the open field test. (E) AMPK-WT and AMPK-cKO mice latency to fall from the rotarod at the same age groups. Statistical analysis: student's two-tailed unpaired t-test ( ${ }^{*} p<0.05$ and ${ }^{* *} p<0.001$ compared to AMPK-WT mice at respective time points, ap $<0.05$ and aap $<0.001$ compared to previous time point within the same group).

A

Figure 3

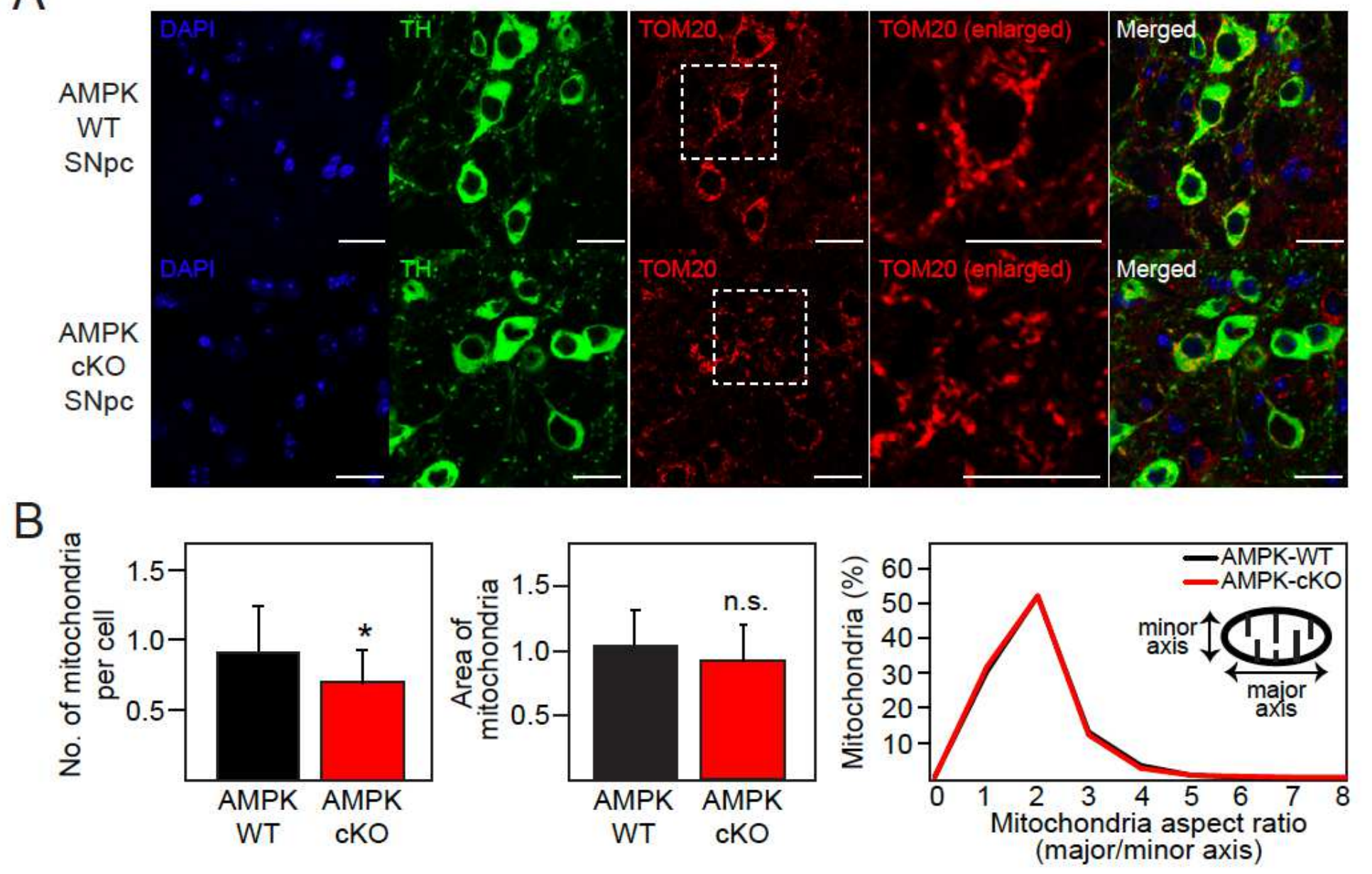

Figure 3

AMPK-cKO mice display aberrations in mitochondrial homeostasis. (A) Representative images showing immunofluorescence labelling of SNpc-containing sections of AMPK-WT and AMPK-cKO mice brains with 
DAPI (blue, nucleus), TH (green) and TOM20 (red, mitochondria). TOM20 staining of the cell located in the white dotted lined box is shown in enlarged view. Merged image shows colocalization of DAPI, TH and TOM20. Scale bar: 20um. (B) Left to right, bar graphs showing the number of mitochondria per cell, average area of mitochondria and line graph showing the distribution of the mitochondria aspect ratios (mitochondria length to width ratios) between nigral TH-positive DA neurons of AMPK-WT and AMPK-cKO mice. Statistical analysis: student's two-tailed unpaired t-test ( ${ }^{\star} \mathrm{p}<0.05$ compared to AMPK-WT).

Figure 4
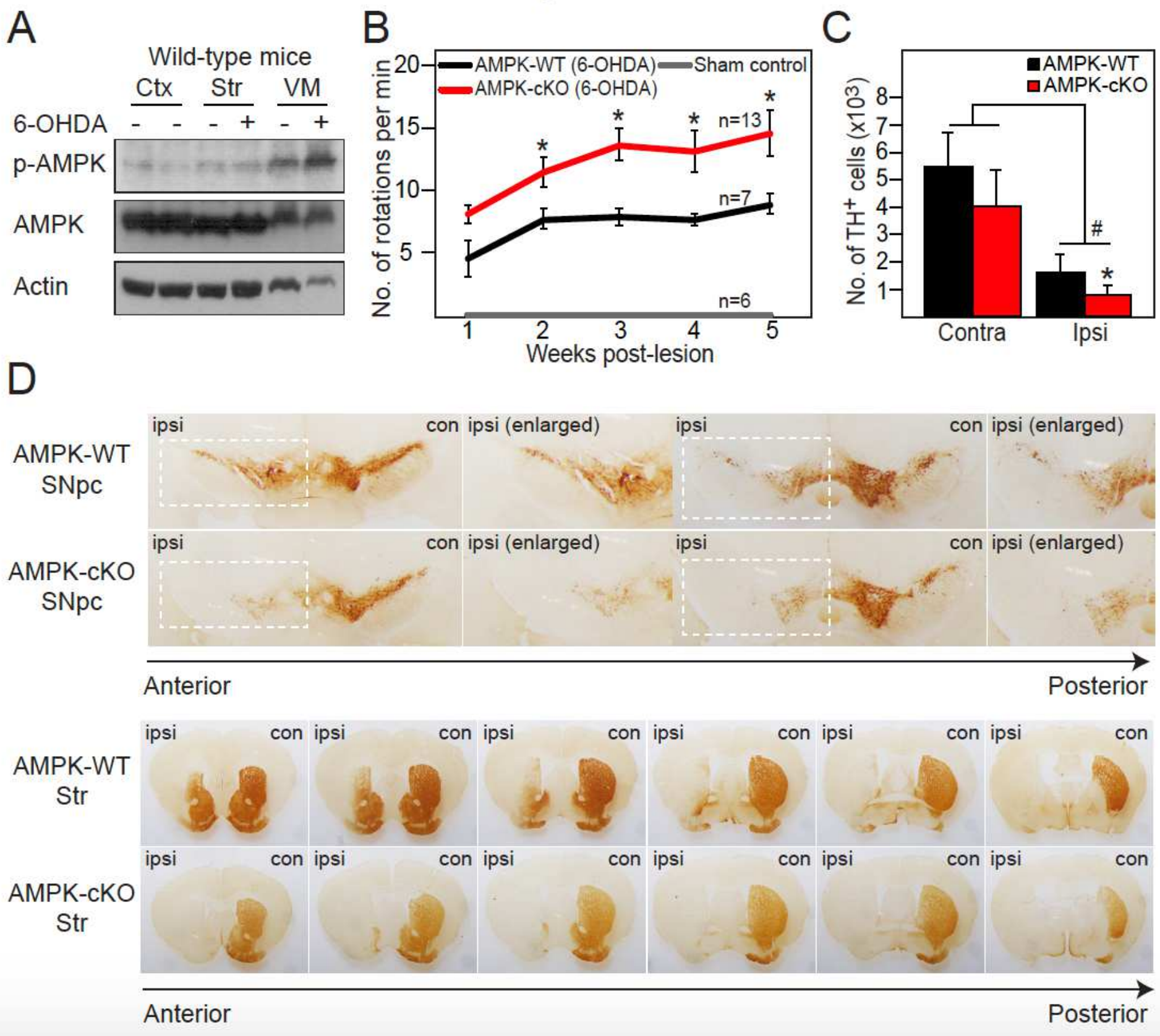

\section{Figure 4}

AMPK-cKO mice are more susceptible to 6-hydroxydopamine (6-OHDA)- mediated toxicity. (A) Representative immunoblots showing the expression of pAMPK, AMPK and actin across selected brain regions (cortex, striatum and ventral midbrain) of wildtype mice in the absence or presence of 6-OHDA. 
(B) Number of apomorphine-induced contralateral rotations of 6-OHDA-lesioned AMPK-WT mice $(n=7), 6$ OHDA-lesioned AMPK-cKO mice $(n=13)$ and sham controls $(n=6)$ from week 1 to week 5 post-surgery. (C) Stereological counts of TH-positive cells in the contralateral (non-lesioned) and ipsilateral (lesioned) sides of brain sections containing SNpc from AMPK-WT or AMPK-cKO mice. (D) Representative immunohistochemical images of the SNpc and striatal (Str) sections of AMPKWT or AMPK-cKO mice five weeks post-surgery, arranged from anterior to posterior position. Positive brown stains indicate presence of TH-positive cells. Statistical analysis: student's two-tailed unpaired t-test $\left({ }^{*} \mathrm{p}<0.05\right.$ compared to AMPKWT mice, \#p<0.05 compared to contralateral side). 